\title{
Effects of dipeptidyl peptidase-4 inhibitor treatment doses on tuberculosis in patients with diabetes: a long-term nationwide population-based cohort study
}

\author{
Hsin-Hung Chen ${ }^{1,2}$, Ming-Chia Hsieh ${ }^{1,3,4}$, Chun-Wei Ho ${ }^{1}$, Ching-Chu Chen ${ }^{5,6}$, Sheng-Pang Hsu ${ }^{5}$, \\ Cheng-Li Lin $^{7,8}$, Chia-Hung Kao, ${ }^{90,11,12}$
}

${ }^{1}$ Intelligent Diabetes Metabolism and Exercise Center, China Medical University Hospital, Taichung; ${ }^{2}$ School of Medicine, Institute of Medicine and Public Health, Chung Shan Medical University, Taichung; ${ }^{3}$ Graduate Institute of Integrative Medicine, China Medical University, Taichung; ${ }^{4}$ Division of Clinical Nutrition, China Medical University Hospital, Taichung; ${ }^{5}$ Division of Endocrinology and Metabolism, Department of Internal Medicine, China Medical University Hospital, Taichung; ${ }^{6}$ School of Chinese Medicine, China Medical University, Taichung; ${ }^{7}$ Management Office for Health Data, China Medical University Hospital, Taichung; ${ }^{8}$ College of Medicine, China Medical University, Taichung; ${ }^{9}$ Graduate Institute of Biomedical Sciences, College of Medicine, China Medical University, Taichung; ${ }^{10}$ Department of Nuclear Medicine and PET Center, China Medical University Hospital, Taichung; ${ }^{11}$ Department of Bioinformatics and Medical Engineering, Asia University, Taichung; ${ }^{12}$ Center of Augmented Intelligence in Healthcare, China Medical University Hospital, Taichung

Contributions: (I) Conception and design: HH Chen, CH Kao; (II) Administrative support: None; (III) Provision of study materials or patients: CH Kao; (IV) Collection and assembly of data: All authors; (V) Data analysis and interpretation: All authors; (VI) Manuscript writing: All authors; (VII) Final approval of manuscript: All authors.

Correspondence to: Chia-Hung Kao, MD. Graduate Institute of Biomedical Sciences, School of Medicine, College of Medicine, China Medical University, No. 2, Yuh-Der Road, Taichung 404. Email: d10040@mail.cmuh.org.tw; dr.kaochiahung@gmail.com.

Background: To investigate the association of dipeptidyl peptidase-4 inhibitors (DPP4is) treatment doses and tuberculosis (TB) in patients with diabetes.

Methods: We allocated participants into DPP4i users and non-users from the Longitudinal Health Insurance Database. A chi-square test and Wilcoxon's rank-sum test were used to analyze the baseline discrete variables and continuous variable, respectively. The incidence rate was calculated in 1,000 personyears. The hazard ratios (HRs) were adjusted using a multivariate Cox regression model. The effect of DDP4i dosage on TB was analyzed. The Kaplan-Meier method was used to assess the cumulative incidence curves with a log-rank test.

Results: We identified 6,399 DPP4i users and 6,399 non-users. The incidence rate of TB in DPP4i users and non-users was 22.2 and 16.2 per 1,000 person-years, respectively. The HR of TB for DPP4i users relative to non-users was $1.04(\mathrm{P}=0.89)$. Most of the analysis of factors such as the incidence rate, gender and diabetic comorbidities in our study were non-significant. The risk of developing TB in patients with over 20 average defined daily doses (DDDs) per year was increased by 2.19 times $(\mathrm{P}=0.048)$.

Conclusions: In our long-term nationwide population-based cohort study, higher doses of DPP4i (20 average DDDs) could increase TB infection risk in patients with diabetes. To pay more attention to this kind of diabetic patients with DPP4i treatment will be more important for the public health issue of TB prevention.

Keywords: Dipeptidyl peptidase-4 inhibitor (DPP4i); tuberculosis (TB); diabetes

Submitted Jan 23, 2020. Accepted for publication Jul 01, 2020.

doi: 10.21037/apm-20-278

View this article at: http://dx.doi.org/10.21037/apm-20-278 


\section{Introduction}

Tuberculosis (TB) caused by Mycobacterium tuberculosis infection remains an infectious disease that causes death in high-risk populations with immunosuppression (1). In 2016, TB infection remained among the 10 most common causes of death, affecting an estimated 1.3 million people globally. The incidence of TB infection is higher in people with undernutrition, diabetes, alcohol misuse, smoking habits, and exposure to indoor air pollution $(2,3)$. Diabetes is an immune dysfunction and metabolic disease that increases the risk of TB infection by three times. Studies have indicated that diabetes is a common comorbidity in patients with $\mathrm{TB}$, and poor outcomes of TB control, such as treatment failure, relapse, and even death, were mostly noted in patients with diabetes $(4,5)$. Diabetes is a common noncommunicable disease globally, and the International Diabetes Federation estimated that the number of people with diabetes will increase to 642 million by 2040. Regarding global public health, the World Health Organization (WHO) suggested bidirectional screening for TB and diabetes (6,7). In 2018, the International Union Against Tuberculosis and Lung Disease and the World Diabetes Foundation published the first guideline for the treatment of patients with TB and diabetes concurrently. This guideline mentioned that patients with newly diagnosed diabetes should be screened for TB in countries, where prevalence is greater than 100 cases per 100,000 patients. In the other hand, all patients with TB should be screened for diabetes. Studies showed that there is a positive association between diabetes and drug-resistant TB possibly because of high initial bacterial loads, slow responses to treatment, and high chances of failing treatment. Metformin remains the first-line drug for treatment of diabetic patients with TB due to the best treatment outcomes (8). Many studies have demonstrated the positive effects of metformin for TB treatment $(9,10)$. DPP4, also known as adenosine deaminase (ADA) complexing protein 2 (CD26), is a $110-\mathrm{kDa}$ surface glycoprotein expressed in numerous cell types with multiple biological functions, including immune activity. CD26 plays a key role in autoimmune regulation and glycemic control through interactions between various cell surfaces and intracellular molecules. Regulating the CD26 receptor could induce different cytokines or chemokines, and the chain reaction might modify DPP4 activity (11).

In our previous series of DPP4i studies, DPP4i appeared to increase infections, such as herpes zoster, but did not increase inflammations, such as allergic rhinitis or chronic rhinosinusitis (12-14). Fewer studies have mentioned the association between TB and DPP4i in patients with diabetes. However, DPP4i are widely prescribed for diabetes control, particularly for elderly patients or those with diabetes and chronic kidney disease (15). This study investigated the effect of DPP4i on TB in patients with diabetes.

We present the following article/case in accordance with the STROBE reporting checklist (available at http://dx.doi. org/10.21037/apm-20-278).

\section{Methods}

\section{Data source}

The data for this study originated from the Taiwan National Health Insurance Research Database (NHIRD), which contains the health information of almost 99\% of Taiwan's population. The Longitudinal Health Insurance Database, which consists of information such as outpatient and inpatient records, medication history, and surgical treatment for 1 million insured patients, was used for analysis. Data on patient identities were scraped cryptographically to protect patient privacy. Disease codes were in accordance with the International Classification of Diseases, Ninth Revision, Clinical Modification (ICD-9-CM).

\section{Study sample}

Patients with diabetes (ICD-9-CM 250.x0 and 250.x2) between 2000 and 2012 were enrolled in the study. We allocated participants into DPP4i users and non-users. The index dates for DPP4i users were the first day of DPP4i treatment, and those for non-users were randomly assigned. Patients receiving a diagnosis of TB before the index date and those less than 20 years old were excluded. We matched DPP4i users and non-users by gender, age, diabetes complications severity index (DCSI) score, and comorbidities at a ratio of $1: 1$. We followed the participants from the index date to the occurrence of TB with antiTB regimens use, loss to follow-up, or the end of the study (December 31, 2013).

\section{Ethics statement}

The study was conducted in accordance with the Declaration of Helsinki (as revised in 2013). The National Health Research Institute (NHRI) of Taiwan is in charge 
of administrating NHIRD, whose personal information of the beneficiaries has been encrypted, and the researchers can apply this database for medical studies. The consents for the patients are exempted in accessing the NHIRD, and the Institutional Review Board (IRB) of China Medical University (CMUH104-REC2-115-CR4) has also approved to waive the consent requirement. The authors are accountable for all aspects of the work in ensuring that questions related to the accuracy or integrity of any part of the work are appropriately investigated and resolved.

\section{Data availability statement}

The dataset used in this study is held by the Taiwan Ministry of Health and Welfare (MOHW). The MOHW must approve our application to access this data. Any researcher interested in accessing this dataset can submit an application form to the MOHW requesting access. Please contact the staff of MOHW (Email: stcarolwu@mohw.gov. tw) for further assistance. Taiwan MOHW address: No. 488, Sec. 6, Zhongxiao E. Rd., Nangang Dist., Taipei 115. Phone: +886-2-8590-6848. All relevant data are within the paper.

\section{Main outcome, comorbidities, and medical treatment}

The primary event of this study was TB (ICD-9-CM $01)$. We considered comorbidities including coronary heart disease (CAD) (ICD-9-CM 410-414), stroke (ICD9-CM 430-438), hypertension (ICD-9-CM 401-405), hyperlipidemia (ICD-9-CM 272), chronic kidney disease (ICD-9-CM 585), chronic obstructive pulmonary diseases (ICD-9-CM 491, 492, 496), and alcohol-related illness (ICD-9-CM 291, 303, 305, 571.0, 571.1, 571.2, 571.3). We also considered medical treatments, namely calcium channel blockers (CCBs), beta blockers, angiotensin II receptor blockers (ARBs), insulin, metformin, and statins as potential confounders.

\section{Statistical analysis}

A chi-square test and Wilcoxon's rank-sum test were used to analyze the baseline discrete variables and continuous variable, respectively. The incidence rate was calculated in 1,000 person-years. A univariate Cox proportional hazard regression model was used to estimate the crude hazard ratio (HR). The HRs were then adjusted by using a multivariate Cox regression model including gender, age, DCSI score, all comorbidities, and all medicines. We calculated the lifetime cumulative dose of DDP4i and standardized the dose of DDP4 $i$ as defined daily dose (DDD) according to the Anatomical Therapeutic Chemical classification system. We further analyzed the doseresponse effect among patients using DDP4i. We calculated the average DDD of DDP4i per year by dividing the total DDD by the follow-up period. The Kaplan-Meier method was used to assess the cumulative incidence curves, which were evaluated using a log-rank test.

\section{Results}

We identified 6,399 DPP4i users and 6,399 non-users, whose baseline characteristics are presented in Table 1. Male and female patients were evenly distributed in the cohort. Participants were mainly $40-59$ years old. The mean age of DPP4i users was $61.9 \pm 13.3$ years, and that of non-users was $62.3 \pm 12.7$ years. Most participants had a DCSI score of $>4$. A higher proportion of DPP4 users had stroke than that of non-users did. Regarding medications, more DPP4i users received $\mathrm{ARB}$, angiotensin-converting-enzyme inhibitors, insulin, metformin, and statin than non-users did. Usage of beta blockers was more common in DPP4i non-users.

As shown in Figure 1, the difference in the cumulative incidences of TB in DPP4 $i$ users and non-users was nonsignificant (log-rank test: $\mathrm{P}=0.2$ ). The incidence rate of TB in DPP4i users was 22.2 per 1,000 person-years, and that in DPP4i non-users was 16.2 per 1,000 person-years (Table 2). The HR of TB for DPP4i users relative to nonusers was 1.04 , which did not reach the level of statistical significance $(\mathrm{P}=0.89)$. Gender and age were nonsignificant factors in TB infection. The effect of DCSI score on TB was also nonsignificant. The relationships of $\mathrm{TB}$ with comorbidities and drug treatment were nonsignificant.

The association of average DDD usage with TB is presented in Table 3. When regarding DPP4i non-users as a reference group, the risk of developing TB in patients with more than 20 average DDDs per year increased by 2.19 times $(\mathrm{P}=0.048)$. Patients with less than 20 average DDDs per year exhibited no effect on TB.

\section{Discussion}

Although the WHO intends to reduce TB incidence by $90 \%$ and mortality by $95 \%$ by 2035 , the incidence of TB decreased by only $2 \%$ per year despite the implementation of directly observed short-course treatment (16). According 
Table 1 Baseline characteristics of diabetes patients with DPP4i or not

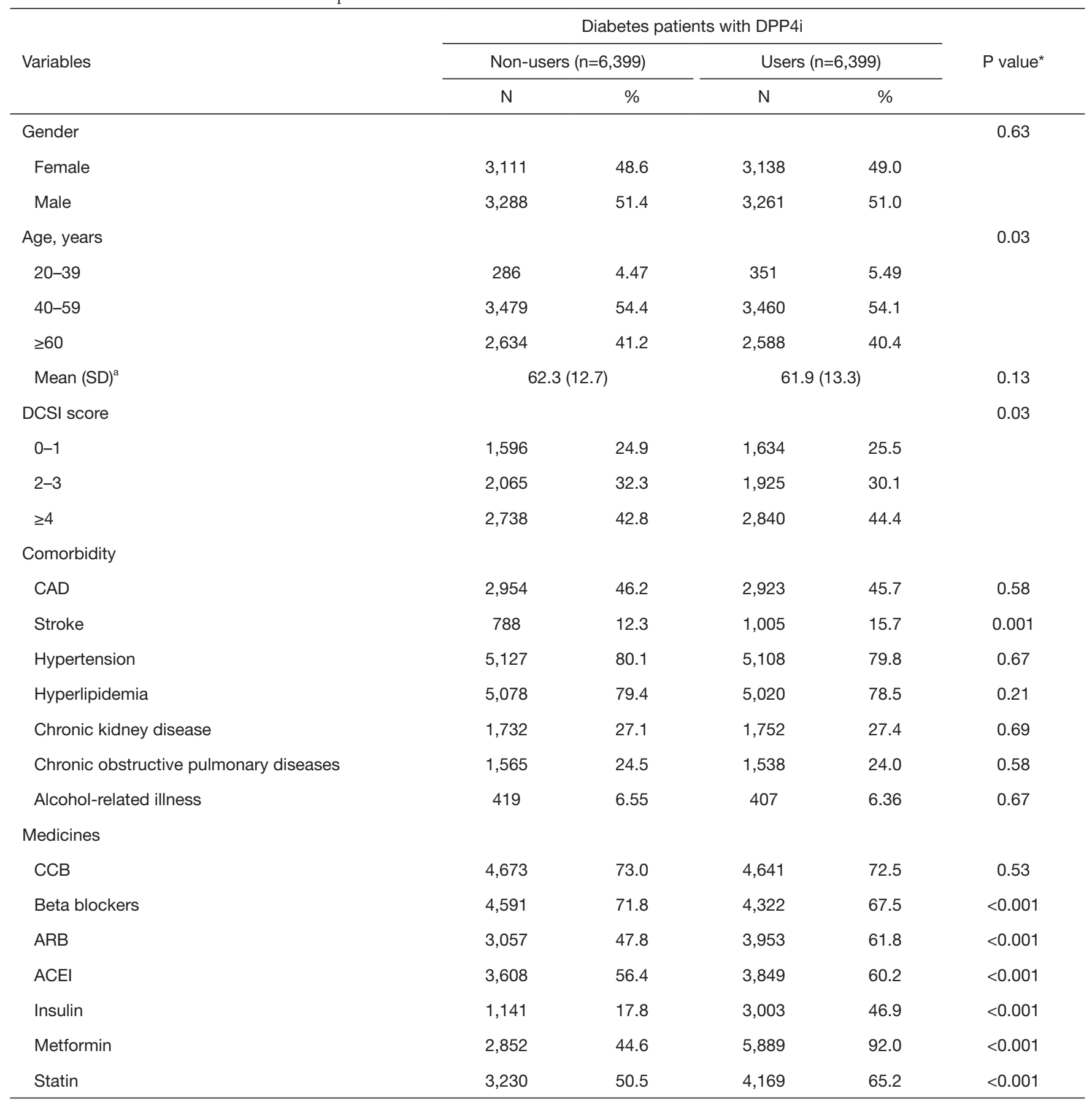

*, Chi-square test; ${ }^{a}$, Wilcoxon's rank-sum test. DPP4i, dipeptidyl peptidase-4 inhibitor; SD, standard deviation; DCSI score, diabetes complications severity index score; CAD, coronary artery disease; CCB, calcium channel blocker; ARB, angiotensin II receptor blocker; ACEl, angiotensin-converting enzyme inhibitor. 


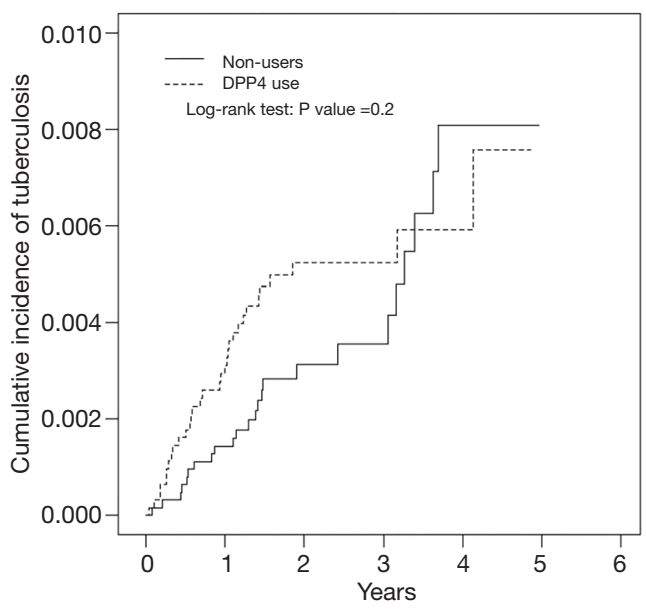

Figure 1 Cummulative incidence of TB between individuals with DPP4i use and without non-users. TB, tuberculosis; DPP4i, dipeptidyl peptidase-4 inhibitor.

to epidemiological research, diabetes is a common risk factor for TB morbidity and mortality in low- and middleincome countries, contributing to the high burden of TB $(17,18)$. The prevalence of diabetes among TB patients ranged from $1.9 \%$ to $45 \%$ and the prevalence of $\mathrm{TB}$ among diabetic patients ranged from $0.38 \%$ to $14 \%$. The highest prevalence of diabetes among $\mathrm{TB}$ patients is observed in Asia. Although the prevalence of TB among diabetic patients is low globally, it is relatively higher in Asia again. The identified risk factors for comorbidity of TB and diabetes were gender, elderly, smoking, poor glycemic control, and family history of diabetes and TB (19). Studies have suggested that metformin use is beneficial for TB treatment in patients with diabetes $(20,21)$. Another study indicated that suitable diabetic control may reduce TB risk (22). International guidelines for diabetes control suggest combination therapy for glycemic control, such as sulfonylureas and DPP4is, after metformin use (15). Few articles have discussed the effects of oral antidiabetic agents apart from metformin for TB control. Systematic reviews of orally active DPP4is, such as sitagliptin and vildagliptin, indicated a significant increase in all-cause infections after sitagliptin rather than vildagliptin therapy $(23,24)$. One review article indicated the immunological mechanisms of diabetes on TB susceptibility including innate immune dysfunction and adaptive immune dysfunction in diabetic patients with the reactions of cytokines from the immune cells. Animal studies showed that diabetes increased the frequency of airway shedding of Mycobacterium tuberculosis even without cavitations and it might be related to a higher pulmonary bacterial load or an alteration in the airway microenvironment in diabetic patients (25). Many DPP4 transcripts were noted in the nasal epithelia of children who suffered from dust mite allergic rhinitis (13). It might be the possible link of DPP4i and TB. DPP4 affects not only glycemic control through incretin regulation but also immune regulation through $\mathrm{T}$ cell activation. DPP4 is present in various organisms, including prokaryotes and eukaryotes. In humans, DPP4 is present in the brush borders of epithelial cells in the kidneys, small and large intestines, liver, and activated leukocytes (T, B, and natural killer cells), and numerous DPP4 transcripts are present in the nasal epithelia of children $(25,26)$. ADA activity is an established marker for diagnosing TB pleuritis. DPP4, also known as ADA complexing protein 2, is a $110-\mathrm{kDa}$ surface-bound ectopeptidase and alternate biomarker. Many studies have demonstrated that interferon (IFN) $-\gamma$ and IFN- $\gamma$-induced protein $10 \mathrm{kDa}$ (IP-10) are also increased at the site of TB pleurisy and are now considered to be additional diagnostic markers $(27,28)$. IP-10, also known as CKCL10, can promote migration of monocytes and $\mathrm{T}$ cells to inflammatory sites. The serum concentration of IP-10 was increased in patients with chronic hepatitis $\mathrm{C}$ virus or $\mathrm{TB}$ infections. One report indicated that inhibiting the host enzyme DPP4 could deactivate IP-10 (29). Another study suggested that CXCL10 antagonism plays a crucial regulatory role in TB pathology. Membrane-bound DPP4 secretion could rapidly deactivate CXCL10, thereby reducing its chemotactic potential, such as Th1 cell and IFN- $\gamma$ functions. The article suggested an unappreciated regulatory role of DPP4 in $\mathrm{TB}$ and that DPP4is may be prescribed as an adjunct immunotherapy for patients with TB (30,31). In our study, higher doses of DPP4is could increase TB infections in patients with diabetes. Well-designed clinical studies or basic animal research are warranted for assessing the direct effect of DPP4i on TB infections. Moreover, diabetes increased sputum culture positivity after 2 months compared with after 6 months of TB treatment, indicating a reduction in the efficacy of TB treatment or an increase in $\mathrm{TB}$ resistance (32). The combination of diabetes and TB is complex for immunity. The positive or negative effects of DPP4i use for patients with diabetes and TB are not as certain as those of metformin use are. A dose-dependent effect of DPP4i was noted in our study. To pay more attention to this kind of diabetic patients with DPP4i treatment will be more important for the public health issue of TB prevention in Asia. 
Table 2 Incidence rate and HR of TB in diabetes patients of DPP4i users compared to non-users

\begin{tabular}{|c|c|c|c|c|c|c|c|c|c|c|}
\hline \multirow{3}{*}{ Variables } & \multicolumn{6}{|c|}{ Diabetes patients with DPP4i } & \multicolumn{4}{|c|}{ Compared to non-user } \\
\hline & \multicolumn{3}{|c|}{ Non-users } & \multicolumn{3}{|c|}{ Users } & \multicolumn{2}{|l|}{ Crude } & \multicolumn{2}{|l|}{ Adjusted } \\
\hline & Event & PY & IR & Event & PY & IR & $\mathrm{HR}(95 \% \mathrm{Cl})$ & $P$ value & $\mathrm{HR}(95 \% \mathrm{Cl})$ & $P$ value \\
\hline Overall & 24 & 14,791 & 16.2 & 32 & 14,397 & 22.2 & $1.36(0.80,2.32)$ & 0.25 & $1.04(0.57,1.92)$ & 0.89 \\
\hline \multicolumn{11}{|l|}{ Gender } \\
\hline Male & 20 & 7,491 & 26.7 & 21 & 7,295 & 28.8 & $1.07(0.58,1.98)$ & 0.82 & $0.83(0.41,1.66)$ & 0.59 \\
\hline \multicolumn{11}{|l|}{ Age } \\
\hline 20-39 & 1 & 718 & 13.9 & 1 & 895 & 11.2 & $0.78(0.05,12.5)$ & 0.86 & $1.62(0.04,70.8)$ & 0.80 \\
\hline $40-59$ & 6 & 8,177 & 7.34 & 11 & 8,155 & 13.5 & $1.85(0.69,5.01)$ & 0.22 & $1.08(0.37,3.12)$ & 0.89 \\
\hline $0-1$ & 7 & 3,897 & 18.0 & 5 & 3,877 & 12.9 & $0.71(0.23,2.23)$ & 0.56 & $0.36(0.10,1.30)$ & 0.12 \\
\hline $2-3$ & 6 & 4,883 & 12.3 & 6 & 4,515 & 13.3 & $1.07(0.34,3.31)$ & 0.67 & $0.72(0.18,2.83)$ & 0.64 \\
\hline$\geq 4$ & 11 & 6,010 & 18.3 & 21 & 6,005 & 35.0 & $1.92(0.93,3.99)$ & 0.08 & $1.66(0.72,3.83)$ & 0.23 \\
\hline \multicolumn{11}{|c|}{ Comorbidity $^{\dagger}$} \\
\hline Yes & 1 & 286 & 34.9 & 31 & 14,110 & 22.0 & $1.32(0.77,2.25)$ & 0.31 & $1.05(0.57,1.92)$ & 0.87 \\
\hline No & 0 & 339 & 0.00 & 24 & 14,453 & 16.6 & - & & - & \\
\hline \multicolumn{11}{|l|}{ Stroke } \\
\hline Yes & 24 & 12,359 & 19.4 & 8 & 2,038 & 39.3 & $1.09(0.38,3.14)$ & 0.88 & $0.78(0.24,2.59)$ & 0.69 \\
\hline No & 7 & 4,141 & 16.9 & 17 & 10,650 & 16.0 & $0.83(0.28,2.47)$ & 0.74 & $0.56(0.16,1.93)$ & 0.36 \\
\hline \multicolumn{11}{|c|}{ Beta blockers } \\
\hline Yes & 9 & 4,774 & 18.9 & 23 & 9,622 & 23.9 & $1.47(0.79,2.76)$ & 0.23 & $1.09(0.52,2.27)$ & 0.82 \\
\hline No & 7 & 4,260 & 16.4 & 17 & 10,531 & 16.1 & $1.15(0.43,3.09)$ & 0.78 & $0.90(0.30,2.69)$ & 0.84 \\
\hline \multicolumn{11}{|l|}{ ARB } \\
\hline Yes & 10 & 5,696 & 17.6 & 22 & 8,700 & 25.3 & $1.43(0.71,2.89)$ & 0.32 & $1.14(0.52,2.50)$ & 0.75 \\
\hline No & 12 & 8,034 & 14.9 & 12 & 6,757 & 17.8 & $1.16(0.50,2.69)$ & 0.73 & $0.86(0.32,2.28)$ & 0.76 \\
\hline \multicolumn{11}{|l|}{ ACEI } \\
\hline Yes & 8 & 5,833 & 13.7 & 24 & 8,564 & 28.0 & $1.65(0.85,3.18)$ & 0.14 & $1.19(0.57,2.47)$ & 0.65 \\
\hline No & 10 & 6,552 & 15.3 & 14 & 8,239 & 17.0 & $0.89(0.35,2.25)$ & 0.80 & $0.72(0.24,2.15)$ & 0.55 \\
\hline \multicolumn{11}{|l|}{ Insulin } \\
\hline Yes & 14 & 7,896 & 17.7 & 18 & 6,500 & 27.7 & $0.75(0.34,1.67)$ & 0.48 & $0.76(0.34,1.73)$ & 0.52 \\
\hline
\end{tabular}

Table 2 (Continued) 
Table 2 (Continued)

\begin{tabular}{|c|c|c|c|c|c|c|c|c|c|c|}
\hline \multirow{3}{*}{ Variables } & \multicolumn{6}{|c|}{ Diabetes patients with DPP4i } & \multicolumn{4}{|c|}{ Compared to non-user } \\
\hline & \multicolumn{3}{|c|}{ Non-users } & \multicolumn{3}{|c|}{ Users } & \multicolumn{2}{|l|}{ Crude } & \multicolumn{2}{|l|}{ Adjusted } \\
\hline & Event & PY & IR & Event & PY & IR & $\mathrm{HR}(95 \% \mathrm{Cl})$ & $P$ value & $\mathrm{HR}(95 \% \mathrm{Cl})$ & $P$ value \\
\hline No & 15 & 12,399 & 12.1 & 9 & 2,392 & 37.6 & $1.46(0.70,3.02)$ & 0.31 & $1.54(0.66,3.62)$ & 0.32 \\
\hline \multicolumn{11}{|c|}{ Metformin } \\
\hline No & 11 & 8,328 & 13.2 & 13 & 6,464 & 20.1 & $0.67(0.09,5.16)$ & 0.70 & $0.58(0.07,4.90)$ & 0.62 \\
\hline \multicolumn{11}{|l|}{ Statin } \\
\hline Yes & 15 & 5,011 & 29.9 & 17 & 9,386 & 18.1 & $1.62(0.70,3.75)$ & 0.26 & $1.03(0.41,2.57)$ & 0.96 \\
\hline No & 16 & 7,552 & 21.2 & 8 & 7,239 & 11.1 & $1.41(0.70,2.85)$ & 0.34 & $1.01(0.44,2.31)$ & 0.99 \\
\hline
\end{tabular}

Models adjusted by gender, age, DCSI score, all comorbidities, and all medicines listed in Table 1. ${ }^{\dagger}$, Patients with any one of comorbidity were classified as the comorbidity group. HR, hazard ratio; TB, tuberculosis; DPP4i, dipeptidyl peptidase-4 inhibitor; PY, person-years; IR, incidence rate, per 1,000 person-years; $\mathrm{Cl}$, confidence interval; DCSI score, diabetes complications severity index score; CAD, coronary artery disease; CCB, calcium channel blocker; ARB, angiotensin II receptor blocker; ACEI, angiotensin-converting enzyme inhibitor.

Table 3 Incidence rate and HR of TB in diabetes patients by average DDD dose per year of DPP4i use compared to non-users

\begin{tabular}{|c|c|c|c|c|c|c|c|c|}
\hline Variables & $\mathrm{N}$ & Event & PY & IR & \multicolumn{2}{|c|}{ Crude } & \multicolumn{2}{|c|}{ Adjusted } \\
\hline Non-users & 6,399 & 24 & 14,791 & 16.2 & 1.0 & & 1.0 & \\
\hline \multicolumn{9}{|c|}{ DPP4i use, average DDD dose per year } \\
\hline$<10$ & 2,709 & 11 & 7,423 & 14.8 & $0.94(0.46,1.93)$ & 0.87 & $0.62(0.29,1.35)$ & 0.23 \\
\hline$>20$ & 2,001 & 12 & 3,132 & 38.3 & $2.17(1.08,4.39)$ & 0.03 & $2.19(1.01,4.77)$ & 0.048 \\
\hline
\end{tabular}

Models adjusted by gender, age, DCSI score, and all comorbidities listed in Table 1. HR, hazard ratio; TB, tuberculosis; DPP4i, dipeptidyl peptidase-4 inhibitor; PY, person-years; IR, incidence rate 100 person-years; CI, confidence interval; DDD, defined daily dose; DCSI score, diabetes complications severity index score.

\section{Limitations}

Our study had several limitations. First, certain laboratory data such as hemoglobin A1C and blood glucose levels, imaging data such as chest X-rays, and sputum or blood cultures were unavailable in the NHIRD. However, we confirmed TB diagnosis by identifying patients with anti-TB regimens. Second, nutritional status such as weight and lifestyle factors such as smoking habits, alcohol consumption, or exercise were unavailable in the NHIRD. However, we attempted to correct the confounders by adjusting for the ICD-9 classifications of chronic obstructive pulmonary disease and alcoholrelated illness.

\section{Conclusions}

In our long-term nationwide population-based cohort study, higher doses of DPP4i use could increase TB infection in patients with diabetes.

\section{Acknowledgments}

Funding: This study is supported in part by Taiwan Ministry of Health and Welfare Clinical Trial Center (MOHW109TDU-B-212-114004), China Medical University Hospital (CMU107-ASIA-19); MOST Clinical Trial Consortium for Stroke (MOST 108-2321-B-039-003). The funders had no role in the study design, data collection and analysis, the 
decision to publish, or preparation of the manuscript. No additional external funding was received for this study.

\section{Footnote}

Reporting Checklist: The authors have completed the STROBE reporting checklist. Available at http://dx.doi. org/10.21037/apm-20-278

Data Sharing Statement: Available at http://dx.doi. org/10.21037/apm-20-278

Conflicts of Interest: All authors have completed the ICMJE uniform disclosure form (available at http://dx.doi. org/10.21037/apm-20-278). The authors have no conflicts of interest to declare.

Ethical Statement: The authors are accountable for all aspects of the work in ensuring that questions related to the accuracy or integrity of any part of the work are appropriately investigated and resolved. The study was conducted in accordance with the Declaration of Helsinki (as revised in 2013). The National Health Research Institute (NHRI) of Taiwan is in charge of administrating NHIRD, whose personal information of the beneficiaries has been encrypted, and the researchers can apply this database for medical studies. The consents for the patients are exempted in accessing the NHIRD, and the Institutional Review Board (IRB) of China Medical University (CMUH104REC2-115-CR4) has also approved to waive the consent requirement.

Open Access Statement: This is an Open Access article distributed in accordance with the Creative Commons Attribution-NonCommercial-NoDerivs 4.0 International License (CC BY-NC-ND 4.0), which permits the noncommercial replication and distribution of the article with the strict proviso that no changes or edits are made and the original work is properly cited (including links to both the formal publication through the relevant DOI and the license). See: https://creativecommons.org/licenses/by-nc-nd/4.0/.

\section{References}

1. Chee CB, Sester M, Zhang W, et al. Diagnosis and treatment of latent infection with Mycobacterium tuberculosis. Respirology 2013;18:205-16.

2. GBD 2016 Causes of Death Collaborators. Global, regional, and national age-sex specific mortality for 264 causes of death, 1980-2016: a systematic analysis for the Global Burden of Disease Study 2016. Lancet 2017;390:1151-210.

3. World Health Organization. Global tuberculosis report 2017. Available online: http://www.who.int/tb/ publications/global_report/en/ (Accessed August 30, 2018).

4. Martinez N, Kornfeld H. Diabetes and immunity to tuberculosis. Eur J Immunol 2014;44:617-26.

5. Baker MA, Harries AD, Jeon CY, et al. The impact of diabetes on tuberculosis treatment outcomes: a systematic review. BMC Med 2011;9:81.

6. IDF DIABETES ATLAS: 7th Edition International Diabetes Federation, 2015. Available online: http://www. idf.org/diabetesatlas (Accessed July 17, 2017).

7. Kapur A, Harries AD, Lönnroth K, et al. Diabetes and tuberculosisco-epidemic: the Bali Declaration. Lancet Diabetes Endocrinol 2016;4:8-10.

8. Mushtaq A Tuberculosis in diabetes: insidious and neglected. Lancet Respir Med 2019;7:483.

9. Lee MC, Chiang CY, Lee CH, et al. Metformin use is associated with a low risk of tuberculosis among newly diagnosed diabetes mellitus patients with normal renal function: A nationwide cohort study with validated diagnostic criteria. PLoS One 2018;13:e205807.

10. Singhal A, Jie L, Kumar P, et al. Metformin as adjunct antituberculosis therapy. Sci Transl Med 2014;6:263ra159.

11. Ohnuma K, Takahashi N, Yamochi T, et al. Role of CD26/ dipeptidyl peptidase IV in human T cell activation and function. Front Biosci 2008;13:2299-310.

12. Li SY, Chen HH, Lai CC, et al. Dipeptidyl peptidase-4 inhibitor treatment could decrease chronic rhinosinusitis in diabetic patients. QJM 2020;113:181-5.

13. Chen HH, Li SY, Chen W, et al. Association between dipeptidyl peptidase-4 inhibitors and allergic rhinitis in Asian patients with diabetes. Int J Environ Res Public Health 2019;16:1323.

14. Chen HH, Lin CL, Yeh SY, et al. Short-term dipeptidyl peptidase-4 inhibitor use increases the risk of herpes zoster infection in Asian patients with diabetes. QJM 2016;109:91-5.

15. American Diabetes Association. 9. Pharmacologic approaches to glycemic treatment: standards of medical care in diabetes-2020. Diabetes Care 2020;43:S98-110.

16. World Health Organization. Global strategy and targets for tuberculosis prevention, care and control after 2015. Available online: http://www.who.int/tb/post2015_ 
TBstrategy.pdf?ua=1 (Cited July 25, 2014).

17. Jeon CY, Murray MB. Diabetes mellitus increases the risk of active tuberculosis: a systematic review of 13 observational studies. PLoS Med 2008;5:e152.

18. Danaei G, Finucane MM, Lu Y, et al. National, regional, and global trends in fasting plasma glucose and diabetes prevalence since 1980: systematic analysis of health examination surveys and epidemiological studies with 370 country-years and 2.7 million participants. Lancet 2011;378:31-40.

19. WorknehMH, BjuneGA, YimerSA.Prevalence and associated factors of tuberculosis and diabetes mellitus comorbidity: a systematic review PLoS One 2017;12:e0175925.

20. Lee MC, Lee CH, Lee MR, et al. Impact of metformin use among tuberculosis close contacts with diabetes mellitus in a nationwide cohort study. BMC Infect Dis 2019;19:936.

21. Tseng CH. Metformin decreases risk of tuberculosis infection in type 2 diabetes patients. J Clin Med 2018;7:264.

22. Lee PH, Fu H, Lai TC, et al. Glycemic control and the risk of tuberculosis: a cohort study. PLoS Med 2016;13:e1002072.

23. Amori RE, Lau J, Pittas AG. Efficacy and safety of incretin therapy in type 2 diabetes: systematic review and metaanalysis. JAMA 2007;298:194-206.

24. Richter B, Bandeira-Echtler E, Bergerhoff K, et al. Dipeptidyl peptidase-4 (DPP-4) inhibitors for type 2 diabetes mellitus. Cochrane Database Syst Rev 2008;(2):CD006739.

Cite this article as: Chen $\mathrm{HH}$, Hsieh MC, Ho CW, Chen CC, Hsu SP, Lin CL, Kao CH. Effects of dipeptidyl peptidase-4 inhibitor treatment doses on tuberculosis in patients with diabetes: a long-term nationwide population-based cohort study. Ann Palliat Med 2020;9(5):2817-2825. doi: 10.21037/apm$20-278$
25. Ayelign B, Negash M, Genetu M, et al. Immunological impacts of diabetes on the susceptibility of mycobacterium tuberculosis. J Immunol Res 2019;2019:6196532.

26. Dinjens WN, ten Kate J, van der Linden EP, et al. Distribution of adenosine deaminase complexing protein (ADCP) in human tissues. J. Histochem. Cytochem 1989;37:1869-75.

27. Nieto-Fontarigo JJ, González-Barcala FJ, San-José ME, et al. Expansion of a CD26low effector TH subset and reduction in circulating levels of sCD26 in stable allergic asthma in adults. J Investig Allergol Clin Immunol 2018;28:113-25.

28. Piras MA, Gakis C, Budroni M, et al. Adenosine deaminase activity in pleural effusions: an aid to differential diagnosis. Br Med J 1978;2:1751-2.

29. Wong PC. Management of tuberculous pleuritis: can we do better? Respirology 2005;10:144-8.

30. Palucci I, Battah B, Salustri A, et al. IP-10 contributes to the inhibition of mycobacterial growth in an ex vivo whole blood assay. Int J Med Microbiol 2019;309:299-306.

31. Blauenfeldt T, Petrone L, Del Nonno F, et al. Interplay of DDP4 and IP-10 as a potential mechanism for cell recruitment to tuberculosis lesions. Front Immunol 2018;9:1456.

32. Kurbatova EV, Cegielski JP, Lienhardt C, et al. Sputum culture conversion as a prognostic marker for endof-treatment outcome in patients with multidrugresistant tuberculosis: a secondary analysis of data from two observational cohort studies. Lancet Respir Med 2015;3:201-9. 